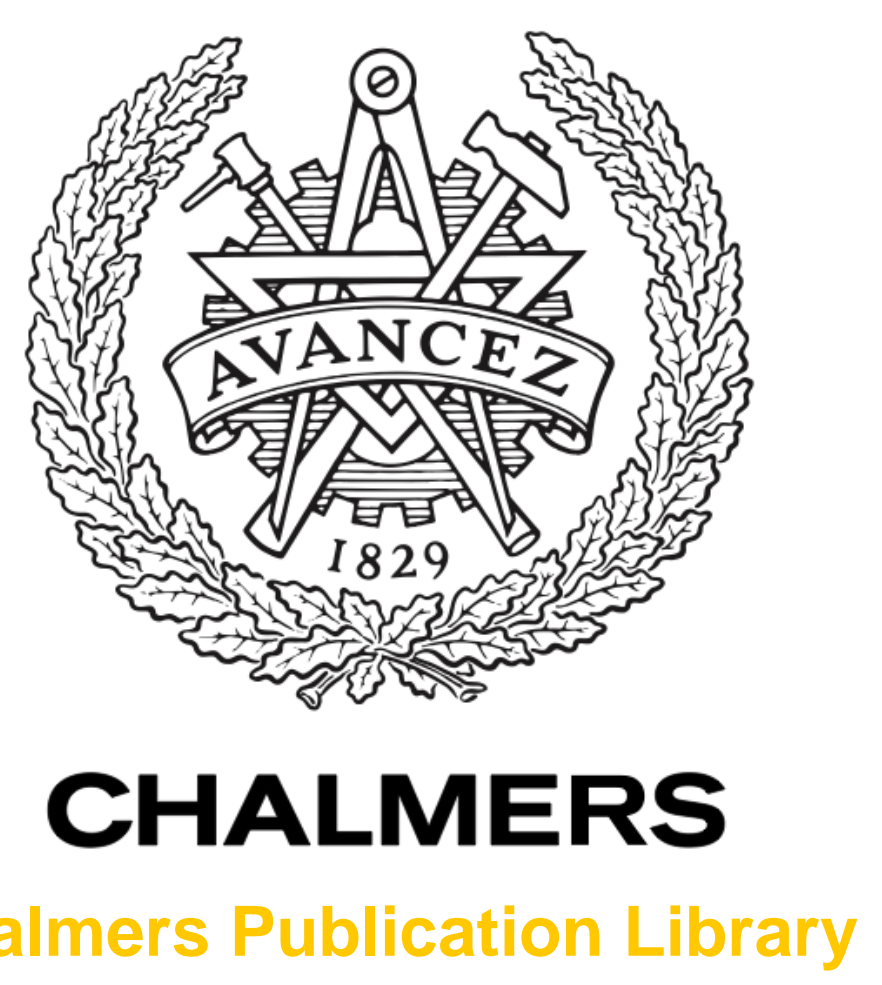

Chalmers Publication Library

\author{
Design, fabrication and measurements with a UV Linear-Variable Optical Filter \\ microspectrometer
}

This document has been downloaded from Chalmers Publication Library $(\mathrm{CPL})$. It is the author's version of a work that was accepted for publication in:

Proceedings of SPIE - The International Society for Optical Engineering, Optical Sensing and Detection II, Brussels,16-19 April 2012 (ISSN: 0277-786X)

Citation for the published paper:

Emadi, A. ; Wu, H. ; De Graaf, G. (2012) "Design, fabrication and measurements with a UV Linear-Variable Optical Filter microspectrometer". Proceedings of SPIE - The International Society for Optical Engineering, Optical Sensing and Detection II, Brussels,16-19 April 2012, vol. 8439

http://dx.doi.org/10.1117/12.922374

Downloaded from: http://publications.lib.chalmers.se/publication/159174

Notice: Changes introduced as a result of publishing processes such as copy-editing and formatting may not be reflected in this document. For a definitive version of this work, please refer to the published source. Please note that access to the published version might require a subscription. 


\title{
Design, fabrication and measurements with a UV linear-variable optical filter microspectrometer
}

\author{
Arvin Emadi ${ }^{* 1}$, Huaiwen $\mathrm{Wu}^{1}$, Ger de $\mathrm{Graaf}^{1}$, Peter Enoksson ${ }^{2}$, Jose Higino Correia ${ }^{3}$ and Reinoud \\ Wolffenbuttel ${ }^{1}$ \\ ${ }^{1}$ Faculty EEMCS, Department ME/EI, Delft University of Technology, Mekelweg 4, \\ 2628 CD, Delft, The Netherlands \\ ${ }^{2}$ Micro and nanosystems, BNSL, MC2 - Chalmers University of Technology - SE-412 96 \\ Gothenburg, Sweden \\ ${ }^{3}$ University of Minho, Department of Industrial Electronics, Campus Azurem \\ 4800 Guimaraes, Portugal
}

\begin{abstract}
An IC-Compatible Linear-Variable Optical Filter (LVOF) for application in the UV spectral range between $310 \mathrm{~nm}$ and $400 \mathrm{~nm}$ has been fabricated using resist reflow and an optimized dry-etching. The LVOF is mounted on the top of a commercially available CMOS camera to result in a UV microspectrometer. A special calibration technique has been employed that is based on an initial spectral measurement on a Xenon lamp. The image recorded on the camera during calibration is used in a signal processing algorithm to reconstruct the spectrum of the Mercury lamp and the calibration data is subsequently used in UV spectral measurements. Experiments on fabricated LVOF-based microspectrometer with this calibration approach implemented reveal a spectral resolution of $0.5 \mathrm{~nm}$.
\end{abstract}

Keywords: Linear Variable Filter, microspectrometer, UV spectrometer

\section{INTRODUCTION}

Ultra-violet (UV) spectroscopy has many applications in pharmaceutical analysis [1], quantitative analysis of transition metals [2], atmospheric gas sensing [3] and bimolecular identification [4]. MEMS-based microspectrometers have huge potential in some of these applications, because of the small sample volume required and the fast response. Additional advantages emerge in case the fabrication of the MEMS-based optical microsystem is compatible with silicon IC technology. On-chip co-integration of the optical system with integrated circuitry enables in-system data pre-processing in the smallest possible system; the chip. Data storage provides opportunities for improving the use-interface and reducing the need for a laboratory with all the logistics associated with such a central infrastructure.

Microspectrometers operating in the UV spectral range require either a small feature size in the grating design, or an optical resonator design with a cavity width less than $200 \mathrm{~nm}$ (when operated in the 300-400 nm band). Fabrication and electronic modulation of a resonator with such a narrow air-gap between the two mirrors is severely hindered by capillary forces inside of the cavity. Also, electrostatic pull-in and subsequent sticking of the two mirrors limits the operating range of the device to one third of the initial air gap [5]-[6].

These problems are avoided in UV Linear Variable Optical Filters (LVOF) fabricated in post-processing steps after completion of the UV photodetector array and circuits in a standard IC technology [6]. Such a LVOF-based microspectrometer was reported for visible spectral range by combining a LVOF with a CMOS camera [7]. In this paper, we present the optical design, implementation and signal processing required for implementing of a LVOF microspectrometer in the UV spectral range. The general basic idea was presented earlier in [8].

Optical Sensing and Detection II, edited by Francis Berghmans, Anna Grazia Mignani, Piet De Moor Proc. of SPIE Vol. 8439, 84390V · (C) 2012 SPIE · CCC code: 0277-786X/12/\$18 · doi: 10.1117/12.922374 


\section{DESIGN AND FABRICATION OF A UV LVOF}

Figure 1 shows a tapered Fabry-Perot type of Linear Variable Optical Filter (LVOF). Collimated light is projected on the surface of the LVOF. The light passing through the LVOF is pass-band determined by the width of the local resonator, and thus by the spatial position along the length of the LVOF [9].

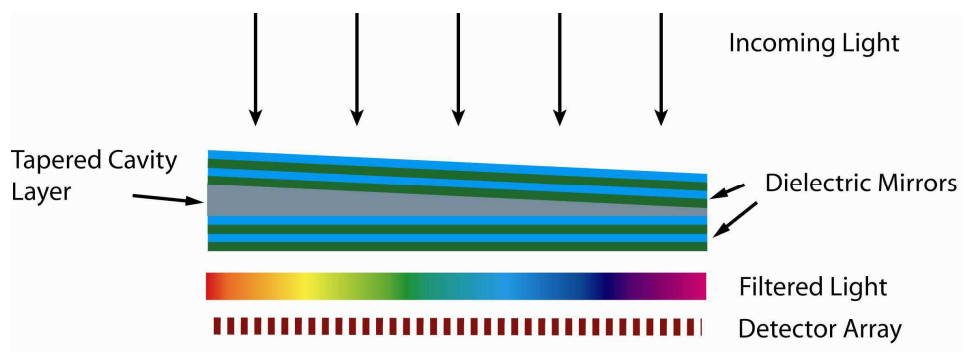

Fig. 1: A schematic view of a tapered Fabry-Perot LVOF

The LVOF is basically a one-dimensional array of many Fabry-Perot (FP)-type of optical resonators. Rather than a huge number of discrete devices, the LVOF has a center layer (the resonator cavity) in the shape of a strip and a thickness that changes over its length. Dielectric mirrors are on either side. Thus the narrow passband wavelength of the LVOF varies linearly along its length. A detector array positioned underneath the LVOF is used for recording the spectrum of the incident light.

The UV LVOF is intended to operate in the wavelength range in between $320 \mathrm{~nm}$ and $400 \mathrm{~nm}$. The filter design critically depends on the choice of the materials used for the high-n and low-n layers. The ratio between the indexes of refraction should be maximum, whereas absorption in the materials should be minimum. A suitable choice for materials in the intended UV spectral range involves $\mathrm{HfO}_{2}$ and $\mathrm{SiO}_{2}$ as respectively high-n and low-n materials. Since the optical parameters of these materials do depend on the details of the deposition technique used, the refractive indexes have been measured by ellipsometry. Table 1 shows the designed thickness of the layers used for the multilayered LVOF, using $\mathrm{HfO}_{2}$ and $\mathrm{SiO}_{2}$. Figure 2 shows the measured refractive indexes of $\mathrm{HFO}_{2}$ and $\mathrm{SiO}_{2}$ in the wavelength range between 280 $\mathrm{nm}$ and $1000 \mathrm{~nm}$. The extinction coefficient in both materials is considered to be negligible for wavelengths above 270 nm.

Table 1. Layers thicknesses of multilayered UV Linear Variable Filter

\begin{tabular}{|l|l|}
\hline Material & Thickness (nm) \\
\hline $\mathrm{HfO}_{2}$ & 43.5 \\
\hline $\mathrm{SiO}_{2} / \mathrm{HfO}_{2}$ & $59 / 43.5$ \\
\hline $\mathrm{SiO}_{2} / \mathrm{HfO}_{2}$ & $59 / 43.5$ \\
\hline $\mathrm{SiO}_{2} / \mathrm{HfO}_{2}$ & $59 / 43.5$ \\
\hline $\mathrm{SiO}_{2} / \mathrm{HfO}_{2}$ & $59 / 43.5$ \\
\hline $\mathrm{SiO}_{2}$ & $440-600$ \\
\hline $\mathrm{HfO}_{2} / \mathrm{SiO}_{2}$ & $43.5 / 59$ \\
\hline $\mathrm{HfO}_{2} / \mathrm{SiO}_{2}$ & $43.5 / 59$ \\
\hline $\mathrm{HfO}_{2} / \mathrm{SiO}_{2}$ & $43.5 / 59$ \\
\hline $\mathrm{HfO}_{2} / \mathrm{SiO}_{2}$ & $43.5 / 59$ \\
\hline $\mathrm{HfO}_{2} / \mathrm{SiO}_{2}$ & $43.5 / 59$ \\
\hline $\mathrm{HfO}_{2}$ & 43.5 \\
\hline
\end{tabular}




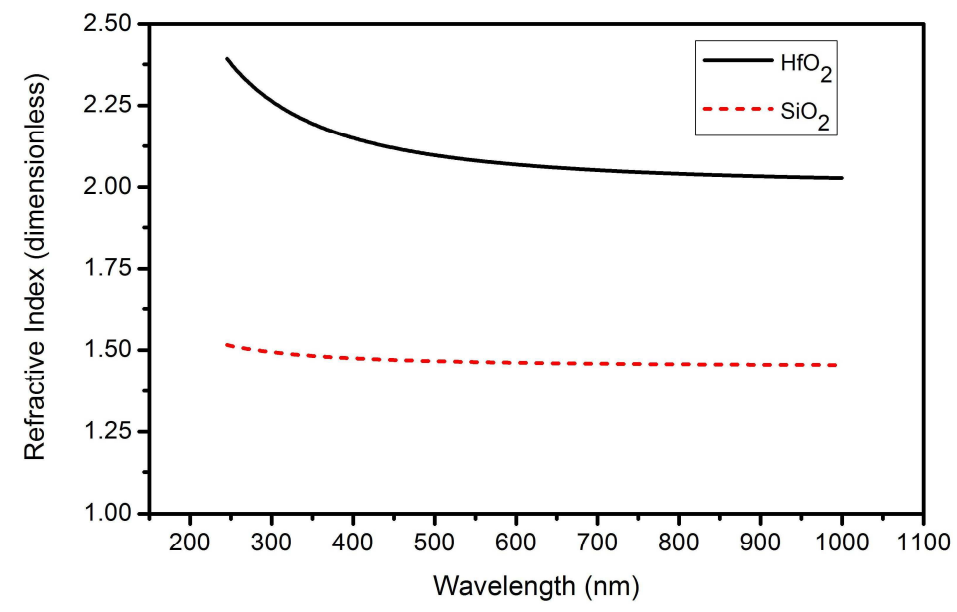

Fig. 2: Refractive indexes of $\mathrm{HfO}_{2}$ and $\mathrm{SiO}_{2}$

The thickness of the tapered cavity layer changes linearly from $440 \mathrm{~nm}$ to $600 \mathrm{~nm}$ to cover the spectral range of interest. The spectral response of the filter is simulated using TFCalc ${ }^{\circledR} 3.3$ and the result is shown in Figure 3.

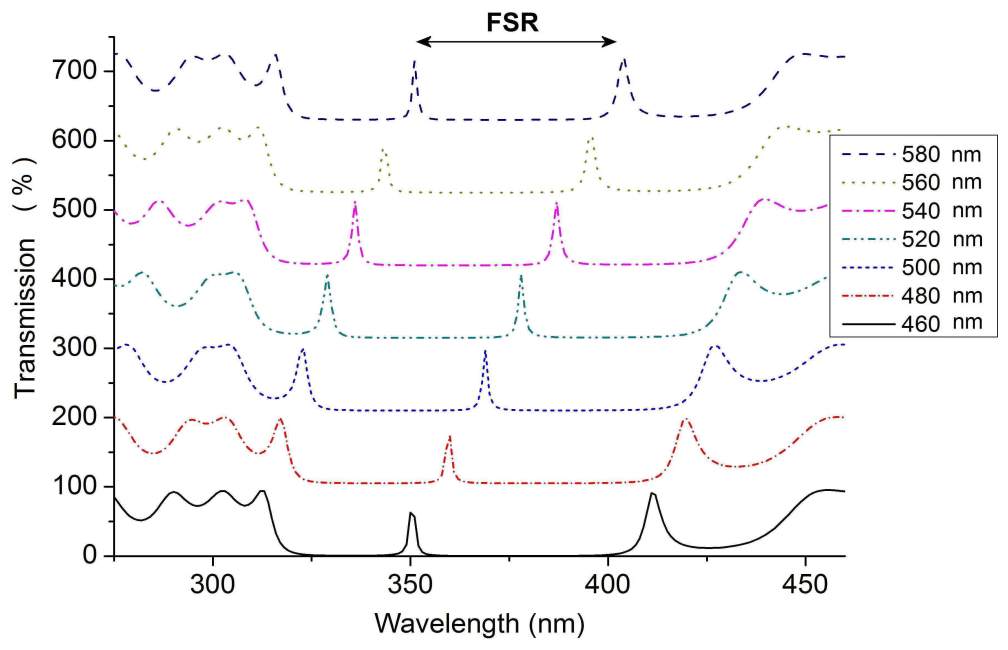

Fig. 3: Simulated spectrum of the LVOF for different values of the cavity thickness

It should be noted that the simulations at the designed values of the layers reveals two different wavelengths that are transmitted through the filter at any value of the cavity thickness (any position along the LVOF). These two wavelengths are from different resonance orders and their wavelength separation limits the Free Spectral Range (FSR). Increasing the thickness of the cavity from $440 \mathrm{~nm}$ to $600 \mathrm{~nm}$ (with transmission as indicted by the curves from bottom to top in Figure 3) covers the spectral range of $315 \mathrm{~nm}$ to $350 \mathrm{~nm}$ in one of the resonance orders, while the other covers the range between $350 \mathrm{~nm}$ and $400 \mathrm{~nm}$.

The maximum reflecting bandwidth of a Bragg reflector, which is the basic element in the structure of the LVOF, can be calculated from Eq. (1). 


$$
\frac{\Delta \lambda}{\lambda_{0}}=\frac{4}{\pi} \operatorname{Arcsin}\left(\frac{n_{2}-n_{1}}{n_{2}+n_{1}}\right)
$$

In which $\Delta \lambda$ is the maximum reflecting bandwidth, $\lambda_{0}$ is the reference wavelength and $n_{2}$ and $n_{1}$ are refractive indexes of the dielectric materials. However, the maximum value of the FSR limits the bandwidth to $\Delta \lambda / 2$. Increasing the thickness of the cavity layer implies a higher operation mode for the Fabry-Perot structure of the LVOF, which results in higher spectral resolution (smaller Full-Width-Half-Maximum, FWHM), but a smaller FSR. For $\mathrm{HfO}_{2}$ and $\mathrm{SiO}_{2}$ and a reference wavelength of $\lambda_{0}=350 \mathrm{~nm}$, the maximum stopband of the dielectric mirrors results from (1) as $\Delta \lambda=84 \mathrm{~nm}$. Consequently, the FSR $=42 \mathrm{~nm}$. The transmission at two different resonance orders has been used for optical characterization of the LVOF and the results are shown in Section 4.

Fabrication involves an IC-Compatible reflow process [10]. Initially, layers 1-10 were sputtered on the substrate. Sputtering of the dielectric layers has been done in the FHR MS 150 sputtering machine. It is possible to achieve an optical thickness variation of the layers less than $2 \%$. Photoresist was spin-coated and patterned by a special pattern optimized to produce a linear slope after reflow. The tapered resist pattern was transferred into a $\mathrm{SiO}_{2}$ cavity layer by a dry-etching process that was optimized for minimum surface roughness.

Figure 4 shows a photograph of the fabricated UV LVOFs. The rainbow pattern on the LVOFs is the result of the slope of the structures. The IC-compatible process for fabrication of the tapered layers allows the possibility for fabrication of devices with a different slope with one lithography step. Because of smaller wavelength in the UV spectrum, as compared to visible spectra, the roughness is more critical in the UV spectral range. The roughness of the surface of the fabricated devices was measured by ellipsometry. Results show a roughness of $4 \mathrm{~nm}$.
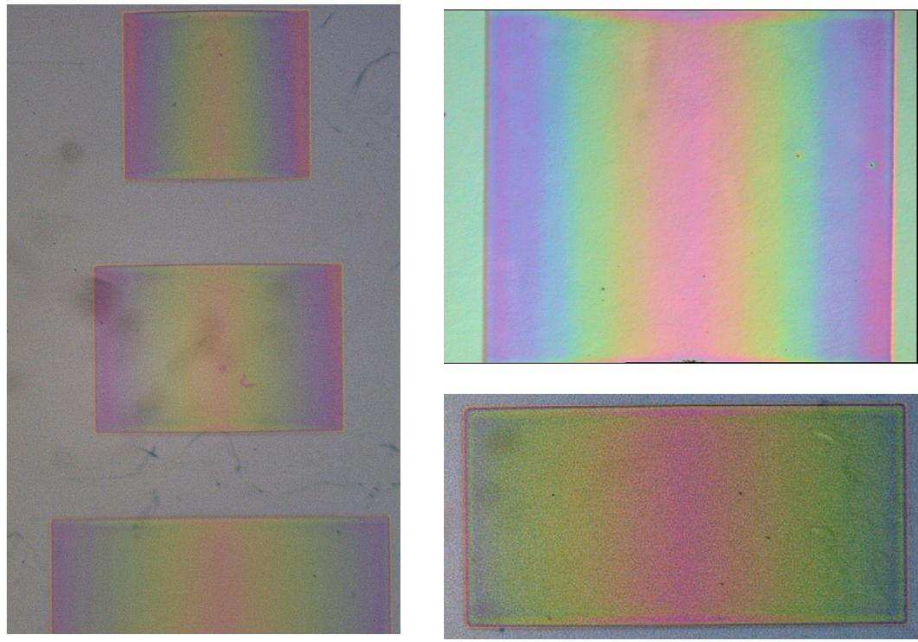

Fig. 4. Photograph of UV LVOFs fabricated on glass substrates.

The structure of an LVOF-based microspectrometer is shown in Figure 5. Light passes an aperture and collimating optics before being projected onto the LVOF, which is placed or deposited on the top of the detector. 


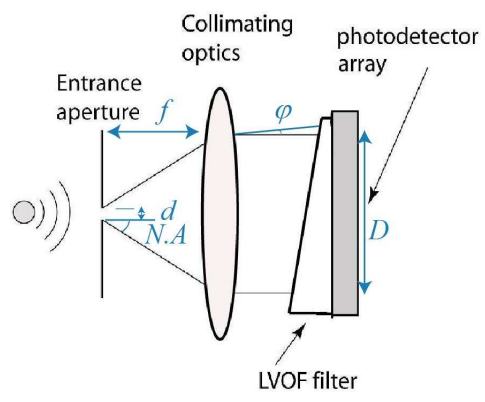

Fig. 5. Structure of a LVOF microspectrometer

From Figure 5 it can be found: $f=\frac{D}{2 \mathrm{NA}}$, in which $\mathrm{D}$ is the length of the LVOF, $f$ is the focal length of the lens and NA is entrance numerical aperture. The Smith-Helmholtz invariant theorem, [11], results in $d \times \mathrm{NA}=D \times \varphi$, which can be rewritten as: $d=D \times \varphi / \mathrm{NA}$. In which, $d$ is the diameter of the aperture and $\varphi$ is maximum acceptable angle of incidence on the LVOF. Since $d$ depends on $\varphi$, the transmission at the position with peak transmission at 349.5 nm simulated for different angles of incidence. The result is that a $5^{\circ}$ deviation from the normal incident the spectral peak of transmission results in a spectral shift over about $0.4 \mathrm{~nm}$. Therefore, the angle of incidence is limited to $\varphi_{\max }=5^{\circ}$ for a maximum spectral inaccuracy of $0.5 \mathrm{~nm}$. The value $\varphi=5^{\circ}$ is inserted in equation (2). Considering a LVOF with a length of $5 \mathrm{~mm}$ and assuming NA $=0.25$ results in $f=\frac{D}{2 \mathrm{NA}}=10 \mathrm{~mm}$ for the focal length of collimating optics. The aperture size is calculated as mentioned and yields: $d=\frac{D \varphi}{\mathrm{NA}}=\frac{5 \mathrm{~mm} \times 5^{\circ}}{0.25 \mathrm{rad}} \approx 1.7 \mathrm{~mm}$. This determines the maximum value of the aperture size, $d$. Therefore, the aperture size in an LVOF-based system is limited by the acceptable spectral shift. A further reduction of aperture improves spectral accuracy at the expense of optical throughput: the amount of light entering the optical system would decrease.

Collimating optics has been designed and implemented in a C-mount holder put on the top of the CMOS camera. Although a C-mount holder is relatively bulky, it is a convenient solution when parameters of collimating optics need to be changed for different experiments. In a final microspectrometer design the C-mount holder has to be replaced with a miniaturized version.

\section{CHARACTERIZATION AND CALIBRATION OF UV LVOF}

Collimated monochromatic light has been projected on the LVOF mounted on the CMOS camera. The wavelength of the projected light changes from $320 \mathrm{~nm}$ to $400 \mathrm{~nm}$ with $0.5 \mathrm{~nm}$ steps, using a programmed monochromator, and results in 161 spectral channels. Figure 6 shows the recorded image on the LVOF spectrometer for different wavelengths in the range from $315 \mathrm{~nm}$ to $400 \mathrm{~nm}$. We can see the reoccurrence of illuminated regions on the images at increment of about $40 \mathrm{~nm}$. This is due to $42 \mathrm{~nm}$ Free Spectral Range (FSR) as explained previously. The recorded intensity profile on the camera pixels is normalized at each wavelength by dividing it to the measured camera responsivity. 


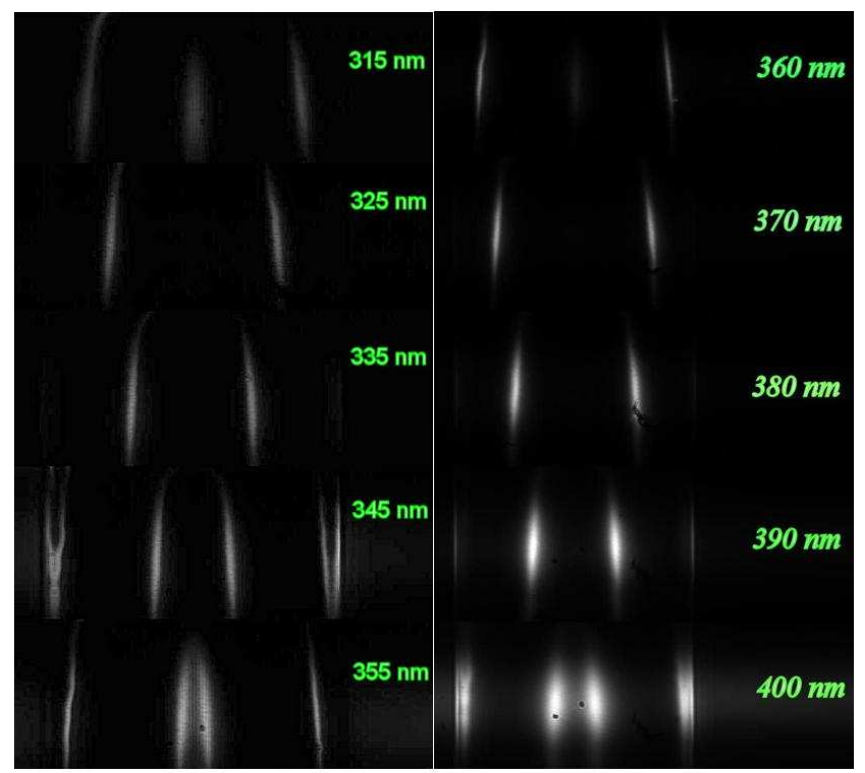

Fig. 6. Recorded image at several wavelengths.

The Half-Power-Line-Width (HPLW) of illuminated regions equals to 9 pixels, which is equivalent to $54 \mu \mathrm{m}$ spatially and $1.2 \mathrm{~nm}$ in terms of resolution. The measured intensity profile recorded by the camera at each wavelength using this monochromator scan is imported (without normalization) into a signal processing algorithm to result in the LVOF microspectrometer calibration matrix, which can subsequently be used for calculation of any spectrum incident on the UV LVOF. The signal processing required is similar to that described in [12] and presented for a visible microspectrometer in [13]. The technique is summarized here.

Let us assume the spectral bandwidth of interest is divided into $\mathrm{N}$ spectral channels and there exists $\mathrm{N}$ spectrally different (independent) detectors. The element $C_{i j}$ in matrix $\mathrm{C}$ is defined as the intensity of channel $i$ of the detector to component $j$ in the spectrum $(i, j=1 \ldots \mathrm{N})$. The matrix $\mathrm{C}$ can be directly constructed from the data of a calibration measurement process. The maximum value of $\mathrm{N}$ is the number of the pixels on the camera, but can be limited by the spectral capability of the calibrating instrument (a monochromator). Hence, the measured intensity on the detector channels can be described as: $D_{1 N}=C_{N N} \cdot I_{1 N}$

In which $d_{i}$ denotes the measured intensity in channel $i$ and $I_{i}$ denotes the input spectrum intensity in channel $i$ that has to be calculated. In other words, Matrix $D_{I N}$ is the raw data recorded on the camera pixels, matrix $I_{I N}$ is the spectrum of light that has to be calculated and matrix $C_{N N}$ is the calibration matrix, which is determined during the calibration process. In the Calibration process, the light from a broadband source (Xenon lamp) is filtered by a monochromator and the selected wavelength is varied in the spectral range of interest for all the $\mathrm{N}$ spectral channels. For each spectral channel from the above equations, calibration is equivalent to deliberately having: $I=\left[0,0, \ldots 0, I_{m}=1,0, \ldots 0\right]$ when channel $m$ is selected from the monochromator. In this case the recorded intensities on the pixels give the values of column $m$ of the C matrix: $D_{1 N}=C_{N N} . I_{1 N}=\left[c_{1 m}, c_{1 m}, \ldots, c_{N m}\right]$.

Repeating the procedure for all the $\mathrm{N}$ spectral channels, results in determination of $C_{N N}$ matrix. For a properly designed and fabricated LVOF, Matrix $C$ has no singularity and it is possible to take the inverse transform of the matrix. Therefore, matrix $I$ can be calculated as $I_{I N}=C_{N N}{ }^{-1} . D_{I N}$. However, since the measured matrix $D$ is added with noise the above solution does not give the best answer. The sources of the disturbance (or noise) in the measured raw data include primarily insufficient collimation, out of the band signal and errors in the estimation of the Calibration matrix (due to inaccuracy in the monochromator instrument). If the disturbance in the system is not negligible as compared to the signal (low SNR) the above approach would result in negative values in some spectral channels, which is not physically acceptable. An iterative procedure needs to be implemented to calculate the matrix $I$ by minimizing matrix $E$ :

$E=D_{1 N^{-}} C_{N N} \cdot \hat{I}_{1 N}$ 
In which matrix $\hat{I}_{N N}$ is the estimate of $I$. The Least Mean Square (LMS) algorithm is implemented based on the following equations:

$$
\begin{aligned}
& E_{n}=d-C \cdot \hat{I}_{n} \\
& \hat{I}_{n+1}=\hat{I}_{n}+\mu C \cdot E_{n}
\end{aligned}
$$

$E_{n} \quad$ denotes the error and $\hat{I}_{n}$ denotes the estimate of the spectrum at each recursive step. $\mu$ is the convergence coefficient. A higher value for $\mu$ results in faster convergence of the algorithm. However, it can also result in divergence (i.e. instability) of the algorithm. The goal of the algorithm is to decrease the mean square of $E_{n}$ at each step. This results in the best possible estimate for the spectrum vector $\hat{I}$. The value of $E_{n}$ remains non-zero, due to noise and disturbances in the measurements.

\section{SPECTRAL MEASUREMENTS}

This LVOF-based microspectrometer is intended for use in the spectral band in between 310 and $400 \mathrm{~nm}$. The total number of channels is 201 each $0.5 \mathrm{~nm}$ wide. The data from the calibration measurement (characterization) is imported into the signal processing algorithm to construct the Calibration matrix, $\mathrm{C}_{\mathrm{NN}}$, as introduced in the previous Section.

The objective is to measure the spectrum of a Mercury lamp in the UV spectral range. The Mercury lamp has a high intensity in this spectral range and demonstrates a unique spectrum. The result is shown in Figure 7 . Two Schott glass filters, types $\mathrm{Ug} 1$ and $\mathrm{Ug} 11$, have been used to eliminate the unwanted part of the spectrum. Ug1 has a passband in 300 $\mathrm{nm}-400 \mathrm{~nm}$ spectral range and Ug11 has the passband in between $250 \mathrm{~nm}$ and $390 \mathrm{~nm}$. As is shown from the measurement with $\mathrm{Ug} 11$, the spectrum for wavelength longer than $380 \mathrm{~nm}$ is eliminated. Therefore, in our measurements with LVOF we have used Ug1 filter to selectively remove the unwanted part of the spectrum.

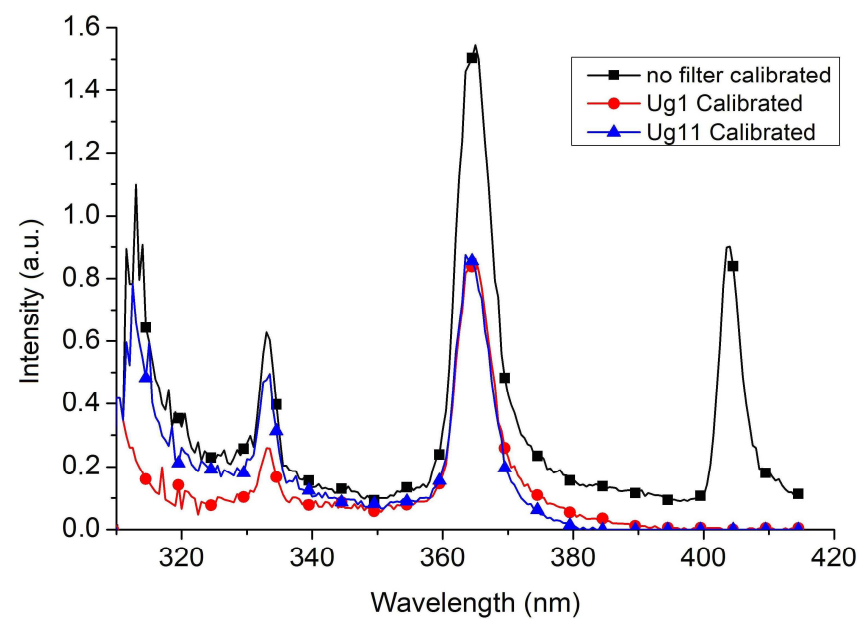

Fig. 7. Measured spectrum of the Mercury lamp, including the effect of the UG1 and UG11 filters.

Figure 8 shows the recorded image on the LVOF camera when illuminated by the Mercury lamp. The image shows three UV LVOFs with different sizes and slopes. We can note the distortions of the bright spectral lines at the sides of the structures, which are due to the reflow process step in the fabrication. This can be prevented in an optimized process. Moreover, data from these parts of the LVOF are neglected in the further calculations. The spectral peaks can be readily identified from the image. 


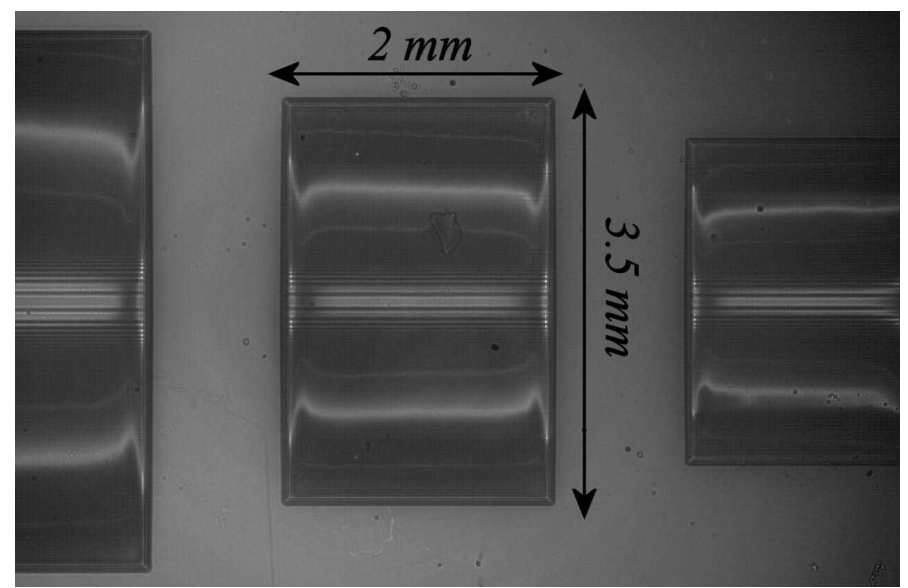

Fig. 8: Image recorded by the UV LVOF microspectrometer when illuminated by a Mercury lamp.

The data from the image is imported to the signal processing algorithm to extract the spectrum. Figure 9 shows a comparison between the spectrum after applying the algorithm and the measurement with a TRIAX 180 monochromator.

The entire spectral range of the LVOF, $320 \mathrm{~nm}$ to $415 \mathrm{~nm}$ has been used in this spectral calculation. From the comparison we can note an extra spectral peak predicted by signal processing algorithm at $385 \mathrm{~nm}$, which is not present in the measurement with the monochromator. This is most probably due to the strong spectral peak of the mercury lamp at $405 \mathrm{~nm}$, which is not completely filtered by the UG1 filter. Considering the much higher sensitivity of the CMOS camera at $405 \mathrm{~nm}$, we can expect that any out-of-band light at higher wavelengths can have a strong negative effect on the performance of the system. The other characteristic difference is the lower calculated baseline of spectral power in the calculated spectrum.

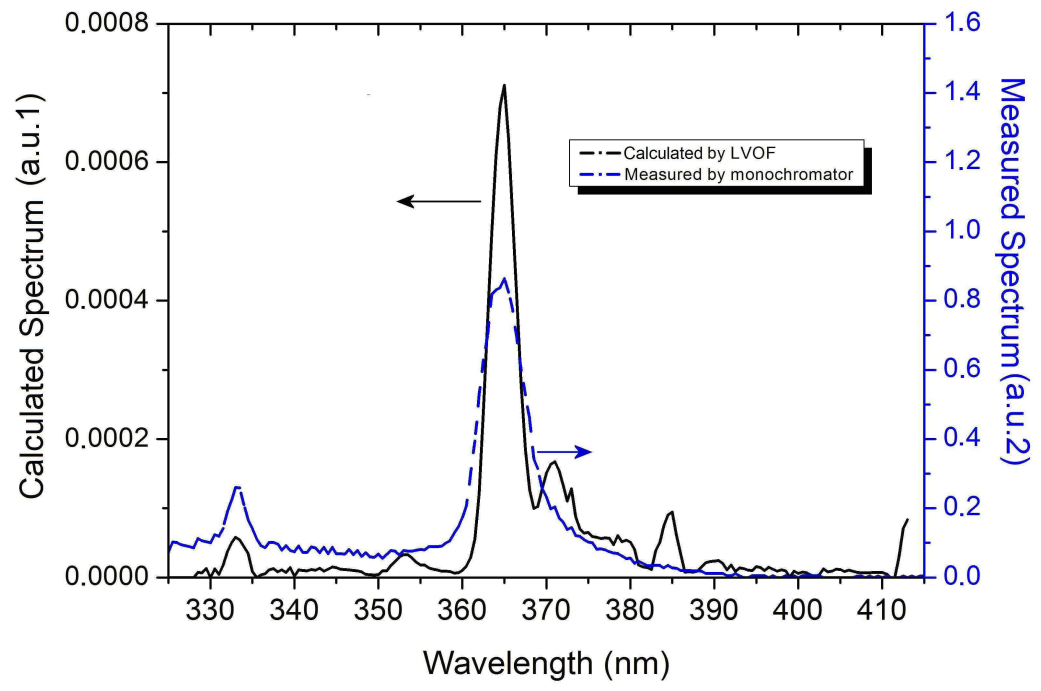

Fig. 9. Calculated spectrum from the LVOF compared with measurement by a monochromator.

The spectral response of the CMOS pixels was taken into account in the calculation. 


\section{CONCLUSIONS}

The design and implementation of a Linear-Variable Optical Filter (LVOF)-based microspectrometer for operation in the UV spectral range is presented in this paper. The IC-Compatible fabrication enables a very compact and rigid microspectrometer system.

The strength of the LVOF-based microspectrometer concept is the high resolving power in a relatively narrow band, which makes it particularly suitable in applications where the spectral analysis around an absorption or emission peak is required, such as in fluorescence. The designed operating range is between $320 \mathrm{~nm}$ and $415 \mathrm{~nm}$. The spectral resolution of the basic microspectrometer is significantly improved by using signal processing to calculate the spectrum of light recorded by the image detector.

First prototypes are bulky because of the C-mount. However, the essential optical path is only $10 \mathrm{~mm} \times 5 \mathrm{~mm}$. The spectral resolution of the basic LVOF is about $1.5 \mathrm{~nm}$ and is improved to $0.5 \mathrm{~nm}$ after application of signal processing.

The most significant limitation at this stage is the low sensitivity of the diodes in the imager in the UV part of the spectrum. The system will be further miniaturized by direct deposition of the LVOF on a customized CMOS imager that is sensitive in the UV range. Collimating optics need also to be miniaturized. The design procedure, fabrication and signal processing of the UV LVOF remain the same.

\section{REFERENCES}

[1] A. Villarisu, M. Fresta, N. Micali, G. Puglisi, Potential application of UV reflection spectroscopy on solid pharmaceutical formulation analysis, International Journal of Pharmaceutics, Volume 127, Issue 2, 17 February 1996, Pages 185-189

[2] A.J. Merer, Spectroscopy of the diatomic 3d transition metal oxides, Ann. Rev. Phys. Chem. 40 (1989), p. 407.

[3] F.Z Chen, D.L Judge, C.Y.Robert Wu, John Caldwell, Low and room temperature photoabsorption cross sections of NH3 in the UV region, Planetary and Space Science, Volume 47, Issues 1-2, 15 December 1998, Pages 261-266

[4] A. Rodger, K. Sanders, Biomacromolecular Applications of UV-Visible Absorption Spectroscopy, In: John C. Lindon, Editor(s)-in-Chief, Encyclopedia of Spectroscopy and Spectrometry, Elsevier, Oxford, 1999, Pages 130-139

[5] L. Mol, L. A. Rocha, E. Cretu and R.F. Wolffenbuttel, "Squeezed film damping measurements on a parallel-plate MEMS in the free molecule regime", J. Micromech. Microeng. 19, (2009).

[6] J. H. Correia, A. Emadi and R. F. Wolffenbuttel, "UV Bandpass optical filter for microspectrometers", ECS transactions, Vol. 4, №1, pp.141-147, Electro Chemical Society, NJ, USA September 2006.

[7] O. Schmidt, P. Kiesel, and M. Bassler, "Performance of chip-size wavelength detectors," Opt. Express 15, 9701-9706 (2007)

[8] A. Emadi, H. Wu, G. de Graaf, K. Hedsten, P. Enoksson, J.H. Correia, R.F. Wolffenbuttel, “An UV linear variable optical filter-based micro-spectrometer," Procedia Engineering, Volume 5, 416-419 (2010).

[9] R. McLeod, T. Honda, Improving the spectral resolution of wedged etalons and linear variable filters with incidence angle, Optics Letters, Vol. 30, No. 19, (2005), pp. 2647-2649.

[10] A. Emadi, H. Wu, S. Grabarnik, G. de Graaf and R. F. Wolffenbuttel, Vertically tapered layers for optical applications fabricated using resist reflow, J. Micromech. Microeng., Volume 19, (2009), pp. 074014/19. 
[11] M. Born, E. Wolf, Principles of Optics: Electromagnetic Theory of Propagation, Interference and Diffraction of Light, 7th ed., (1999).

[12] D. Massicotte, R.Z. Morawski, A. Barwicz, Kalman-filter-based algorithms of spectrometric data correction-Part I: an iterative algorithm of deconvolution, Instrumentation and Measurement, IEEE Transactions on IM, Volume 46, Number 3, (1997), pp. 678-684

[13] A. Emadi, H. Wu, Ger. De Graaf, and R.F. Wolffenbuttel, "Design and implementation of a sub-nm resolution microspectrometer based on a Linear-Variable Optical Filter", Accepted for publication in Optics Express on November 2011. 